\title{
REVIEW
}

\section{Ecotin: Lessons on survival in a protease-filled world}

\author{
MARY E. MCGRATH, ${ }^{1}$ SARAH A. GILLMOR, ${ }^{2}$ AND ROBERT J. FLETTERICK ${ }^{3}$ \\ ' Khepri Pharmaceuticals, Inc., South San Francisco, California 94080 \\ 2 Graduate Group in Biophysics, University of California, San Francisco, California 94143-0448 \\ ${ }^{3}$ Department of Biochemistry and Biophysics, University of California, San Francisco, California 94143-0448
}

(ReceIved October 26, 1994; ACCEPTED November 29, 1994)

\begin{abstract}
Ecotin, an Escherichia coli periplasmic protein of 142 amino acids, has been shown to be a potent inhibitor of a group of homologous serine proteases with widely differing substrate recognition. It is highly effective against a number of enzymes, including both pancreatic and neutrophil-derived elastases, chymotrypsin, trypsin, factor $\mathrm{Xa}$, and kallikrein. Recent structural and functional studies on ecotin and its interactions with different serine proteases have clarified these initial observations and revealed the remarkable features of this protein in inhibiting a strikingly large subset of the chymotrypsin family of serine proteases. The structures of the ecotin:serine protease complexes provide the first examples of protein-protein recognition where the concept of specificity of interactions needs to be reexamined. The binding sites show a fluidity of protein contacts derived from ecotin's innate flexibility in fitting itself to proteases while strongly interfering with their function.
\end{abstract}

Keywords: allostery; crystallography; inhibitors; protein engineering; protein-protein interactions

Thousands of small (25-220 amino acids) inhibitors of serine proteases have been identified from mammals, birds, reptiles, plants, and microorganisms. Their existence reflects the essential roles serine proteases play in processes such as digestion, coagulation, fertilization, development, and invasion. A great number of the inhibitors are unrelated in primary structure and overall topology. However, they have converged upon the motif of an extended surface loop emanating from the core of the protein, which bestows inhibitory activity by binding to the target protease in a substrate-like manner. These inhibitors are generally specific for a particular protease in that the sequence along this "reactive site" loop is complementary to the substrate specificity of the protease. Thus, a common strategy is for an organism to make a family of inhibitors that differ (perhaps) only in their $\mathrm{P} 1$ amino acid, whose side chain will bind in the primary specificity pocket of the protease (Laskowski \& Kato, 1980). High-resolution X-ray structures exist for a number of protease:inhibitor complexes, such as trypsin complexed with an inhibitor from squash (Bode et al., 1989), elastase bound to an Ascaris inhibitor (Huang et al., 1994), and kallikrein with pancreatic trypsin inhibitor (Chen \& Bode, 1983). Although some inhibitors show limited affinity for more than one protease, the fit between protease and inhibitor is generally so exquisite, and the specificity so limited, that the inhibitor appears to be custom-

Reprint requests to: Robert J. Fletterick, Department of Biochemistry and Biophysics, University of California, San Francisco, California94143-0448; e-mail: flett@msg.ucsf.edu. designed for a particular protease. What then are the secrets of a molecule that demonstrates extremely potent inhibition of a mélange of serine proteases that vary significantly in their specificities?

The molecule is ecotin. It is an efficacious inhibitor of trypsin, chymotrypsin, and elastase (Chung et al., 1983) and has inhibition constants in the picomolar range for disease-associated proteases such as factor Xa (Lauwereys et al., 1993) and human leukocyte elastase (Seymour et al., 1994). It also strongly inhibits proteases as diverse as kallikrein, urokinase, factor XIla, fiddler crab collagenase, and granzyme B (C. Bleackley, pers. comm.; Seymour et al., 1994; Tsu et al., 1994). The strength of inhibition does not appear to be correlated with a particular protease specificity.

Surprisingly, the initial report of a molecule from the Escherichia coli periplasm that strongly inhibited the pancreatic serine proteases (Chung et al., 1983) did not inspire a swarm of experiments to further characterize ecotin. It was determined to be a dimer capable of binding one or two protease molecules and demonstrated characteristics common to protease inhibitors: it was relatively small and highly stable and could be purified almost to homogeneity by boiling (Chung et al., 1983). Ecotin was cloned using PCR with peptide sequence information derived from mass spectrometry (McGrath et al., 1991b). The gene codes for a 142-amino acid monomer (Fig. 1) with an implied 20-amino acid signal peptide necessary to direct it to the periplasmic space (McGrath et al., 1991b). The sequence does not resemble that of any known inhibitor or any other gene prod- 
Ecotin Sequence and Composition

\section{Signal Peptide}

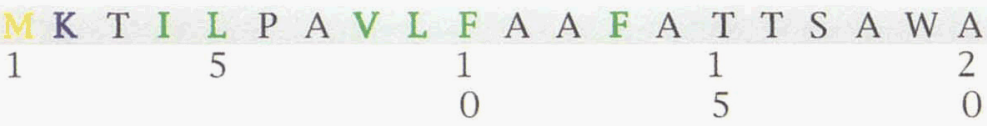

$\begin{array}{llllllllllllllllllll}\text { A } & E & S & V & Q & P & L & E & K & I & A & P & Y & P & Q & A & E & K & G & M\end{array}$

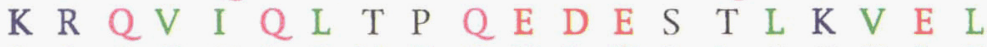

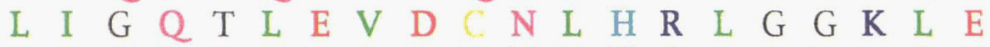

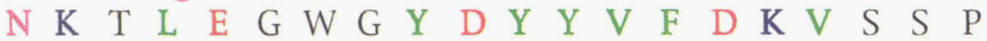
P4 P3 P2 P1 P1' P2' P3' P4'

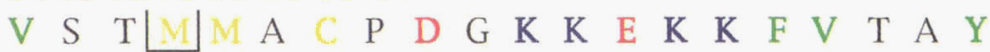

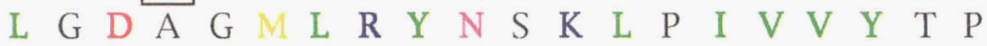

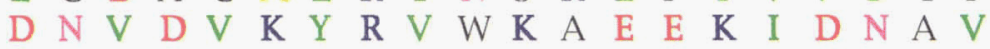
V R
Fig. 1. Amino acid sequence and amino acid composition deduced for ecotin. The columns labeled P4-P4' represent the portion of the reactive site that is expected to engage most proteases. The $\mathrm{P} 1$ residue, Met 84 , is a compromise residue that complements the primary specificity site (S1) of the serine proteases.

\section{Composition:}

$\begin{array}{llll}\text { A: } 8 & \text { C: } 2 & \text { D: } 9 & \text { E: } 12 \\ \text { F: } 2 & \text { G: } 9 & \text { H: } 1 & \text { I: } 5 \\ \text { K: } 15 & \text { L: } 13 & \text { M: } 4 & \text { N: } 5 \\ \text { P: } 8 & \text { Q: } 6 & \text { R: } 5 & \text { S: } 6 \\ \text { T: } 7 & \text { V: } 15 & \text { W: } 2 & \text { Y: } 8\end{array}$

uct. The formal charge based on amino acid composition is neutral (Fig. 1). Yet, ecotin will equally inhibit proteases with a surplus of positive charges (cow cationic trypsin) or an excess of 19 negative charges, as found in collagenase (Tsu et al., 1994). Subsequent cloning by an independent group confirmed the primary structure (Lee et al., 1991). The position of the eco gene was mapped on the E. coli chromosome (Erpel et al., 1992) in order to facilitate (still incomplete) genetic studies that might illuminate ecotin's definitive role in its host bacterium.

Ecotin's reactive site, specifically the P1 residue, was determined for its complexes with trypsin, chymotrypsin, and elastase (McGrath et al., 1991b), and more recently factor Xa (Lauwereys et al., 1993; Seymour et al., 1994), and was found to be Met 84 in all cases. Identification of the reactive site allowed alignment of ecotin with other families of small, substratelike serine protease inhibitors. The reactive site loop of ecotin is similar to those of bovine pancreatic trypsin inhibitor (BPTI), soybean trypsin inhibitor (STI), turkey ovomucoid inhibitor, and others, in many ways, but also features some interesting differences. For example, there is a cysteine that forms a disulfide with an inner supporting loop in these inhibitors. However, in ecotin this residue is positioned at $\mathrm{P} 3^{\prime}$, whereas it is located on the aminoterminal side of the potential scissile bond, at P2 or P3, in the other molecules. Ecotin's Thr at P2 is a frequent choice, as is Pro distal at P4'. Met 85 of ecotin is unusual at P1'. Perhaps most interesting, though, is P1. Good inhibitors of trypsin typically have Arg or Lys at P1: ecotin's Met 84 is an anomaly. However, it is not the only one. Streptomyces subtilisin inhibitor (SSI) (Takeuchi et al., 1991) and $\alpha 1$-antitrypsin (Laskowski \& Kato, 1980) are other inhibitors that have more relaxed specificities and are able to inactivate trypsin using Met at P1. Thus, Met emerges as a suitable compromise residue for satisfying the steric constraints of different protease substrate-binding pockets, and forms the basis for at least part of ecotin's properties.

The desire to illuminate ecotin's mechanism of action through $X$-ray diffraction studies motivated attempts to overexpress the inhibitor in E. coli. Ecotin was easily expressed to greater than $400 \mathrm{mg} / \mathrm{L}$ of $E$. coli culture using its endogenous signal peptide with production controlled by the double tac promoter (McGrath et al., 1991a). It has also been expressed to high levels using a derivative of the phGH1 vector (Seymour et al., 1994) and the pT7-7 vector (Pal et al., 1994).

Ecotin was cocrystallized with a well-characterized mutant of rat anionic trypsin (McGrath et al., 1991a), as well as by itself (Shin et al., 1993). Because no suitable structural homolog of ecotin existed to allow molecular replacement, the strategy for structure determination was to use trypsin as the search model. Thus, the model represented a mere $30 \%$ of the scattering matter in the cell. Molecular replacement was successfully carried out, including the steps of self rotation and cross rotation searches, and Patterson correlation refinement (Brünger, 1990) to facilitate the subsequent translation search. The set of phases that represented the "answer" in this experiment yielded an electron density map that was reasonable for the trypsin part of the complex, but difficult to interpret for the unknown ecotin moiety. Biochemical experiments provided some fiducial points for building in the protein backbone because the amino acid resident in the trypsin binding pocket was known to be Met 84 , and a disulfide was believed to be present between Cys 50 and Cys 87 . A protocol for phase refinement by skeletal modification (PRISM) was being developed (Baker et al., 1993) and appreciably improved the electron density for ecotin (Bystroff et al., 1993). The 
model was then successfully built and refined (McGrath et al., 1994).

Structure determinations have subsequently been carried out for ecotin alone (this structure is preliminary and currently being refined) (S.W. Suh \& D.H. Shin, pers. comm.), ecotin complexed with chymotrypsin (C. Cambillau, pers. comm.), and ecotin bound to fiddler crab collagenase (J.J. Perona, pers. comm.). These structures were also solved by molecular replacement using coordinates from the ecotin:trypsin structure as the basis for a search model. All structures show that ecotin's topology is closely related to the jelly-roll motif, a structure that can be thought of as a sandwich of two sheets. Ecotin's fold differs from a typical jelly-roll in that one strand is missing and two strands are interchanged. With the strands labeled $C$ through $\mathrm{H}$, strand $\mathrm{E}$ rather than strand $\mathrm{F}$ of the second sheet lies closest to the hinge region between the sheets. The position switch for the two strands places strand $\mathrm{E}$ parallel with strand $\mathrm{H}$, contrary to the all-antiparallel nature of jelly-roll fold proteins. The secondary structure is presented in Figure 2.

The $\mathrm{P} 1$ residue (Met 84 ) of ecotin extends from a surface loop, similar locally in structure to the reactive site residues of other small serine protease inhibitors such as BPTI (Ruhlmann et al., 1973), STI (Sweet et al., 1974), and turkey ovomucoid inhibitor third domain (Fujinaga et al., 1987). As with many other small serine protease inhibitors, a disulfide bond (Cys 50-Cys 87) proximal to the reactive site in ecotin stabilizes the reactive site loop by covalently anchoring it to an inner supporting loop (Bode \& Huber, 1992). Recent experiments in which the two cysteines were replaced with alanines (C.S. Craik, pers. comm.) or where Cys 87 was replaced with Ser (Seong et al., 1994) have demonstrated that the disulfide is not absolutely required for the inhibitory potency of ecotin, but may be necessary for thermal stability. Numerous hydrogen bonds between the inner and outer loops (especially residues Thr 83 [P2] and Ser 82 [P3] and also Asn 51) provide additional support. In ecotin, residues 89-92 of the outer loop extend away from the inner loop, making few contacts. This region can be disordered or highly flexible even in intimate complexes with protease (C. Cambillau, pers. comm.; McGrath et al., 1994).

\section{Ecotin's protein-protein interactions}

\section{Ecotin's interactions with itself}

Ecotin dimerizes through its extended C-terminal arm, with the two monomers in a head to tail arrangement. Ten hydrogen bonds exist between the monomers involving residues 125 through the carboxy-terminal residue 142 . The structure formed

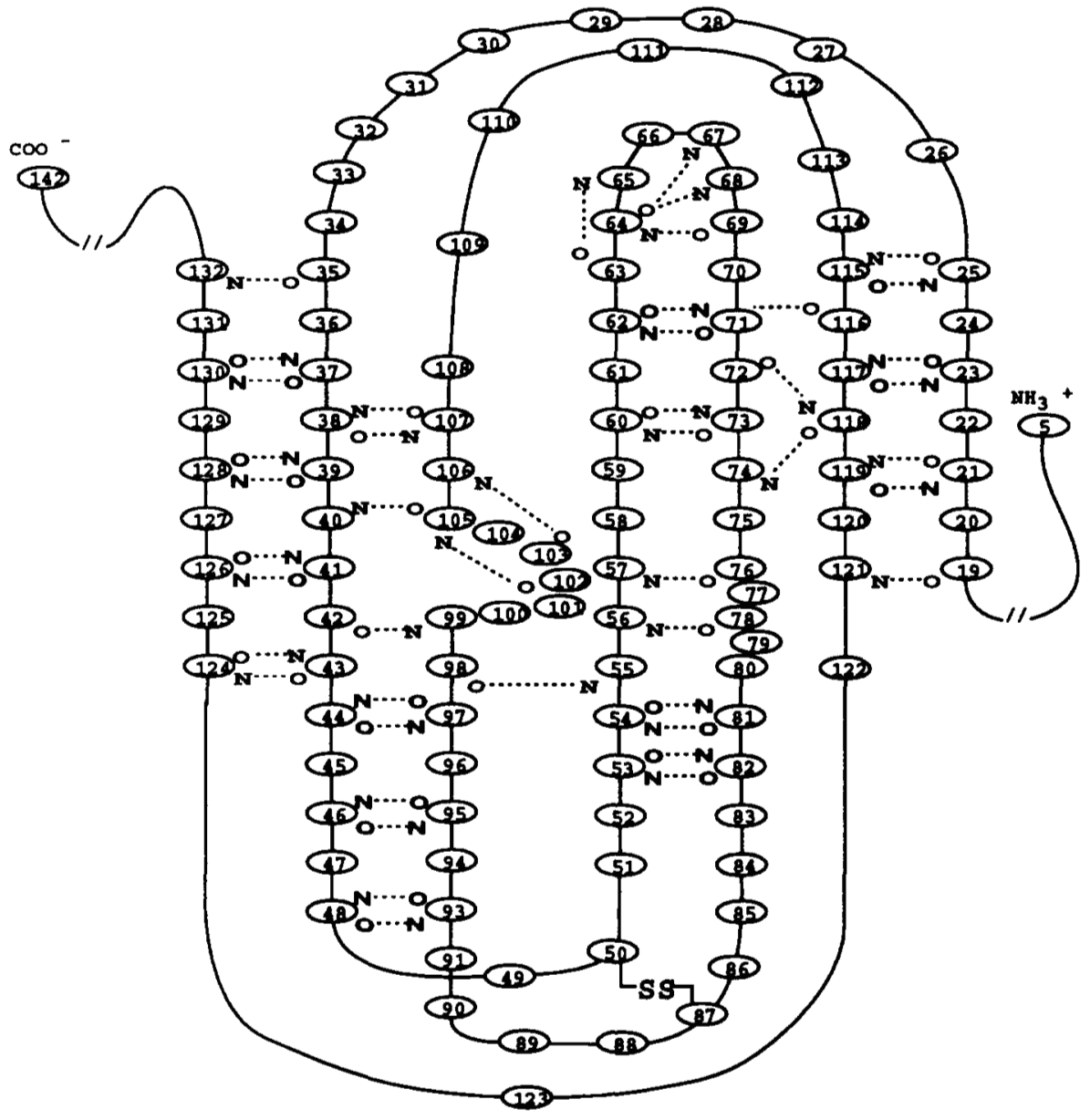

I $\quad$ D $\quad$ G
$\mathbf{E}$
Fig. 2. Secondary structure of ecotin. Although the C-terminus from 133 to 142 interacts with the the dyad-related subunit, the $\mathrm{N}$-terminus is disordered from residues 1 to 4 . The $\beta$-strands form a pair of sheets with the jelly-roll notation shown at the bottom. Strands A and B are not present in this modification, and strands $E$ and $G$ are parallel. The bulge at 102 abuts its dyad mate to form an isolated intersubunit contact on the face opposite the C-terminal embrace. 
at that site is a two-stranded antiparallel $\beta$-ribbon. A $\beta$ bulge at residues 133-134 causes the $C$-terminal arm to terminate with residues $139-142$ positioned orthogonally with respect to the core sheet. The surface area buried when monomers associate is greater than 2,800 $\AA^{2}$ (Fig. 3). This extended area is typical of a structural interface, where extensive surface contacts are necessary for stabilizing monomers in an oligomeric protein (Janin $\&$ Chothia, 1990). The dimer dissociation constant has been determined by fluorescence titration (Seymour et al., 1994) and by microcalorimetry (M. Doyle, pers. comm.) as approximately 390 $\mathrm{nM}$ in the absence of protease. The four ecotin structures show that, although there is some structural adaptation of ecotin to the features of the bound protease, the dimerization region is invariant (Fig. 4). However, comparison of the ecotin:trypsin and ecotin:collagenase structures shows that the angular position and separation of the monomers is adjustable to a few degrees and $1 \AA$ (J.J. Perona, pers. comm.).

\section{Ecotin's primary interactions with proteases}

In the three structures of ecotin bound to protease, Met 84 is the $\mathrm{P} 1$ residue. Like other inhibitors of the canonical class, ecotin forms a distorted Michaelis complex with the protease (Ruhlmann et al., 1973; Perona et al., 1993). There is a sub-van der Waals contact between Met 84-C and the nucleophilic Ser 195 -
$\mathrm{O} \gamma$ of the protease. Met 84-O faces the oxyanion hole (Henderson et al., 1972) and forms hydrogen bonds with the amides of Ser 195 and Gly 193. The side chain of Met 84 is found in three different conformations in the substrate binding pocket, as dictated by the varied shapes and characteristics of these pockets in the three proteases (Fig. 5). The side chain is found in an extended conformation in the trypsin pocket as it mimics the conformation of a preferred Lys. To date, this structure is the only one in which bound solvent has been refined. Similar to the structure with BPTI featuring Lys at P1 (Bode \& Schwager, 1975), six water molecules are found in the binding pocket. In fact, the water molecule that mediates the interaction between Lys-N $\zeta$ and trypsin's Asp 189 side chain has a homolog in the ecotin:trypsin complex structure. In the complex with chymotrypsin, Met 84 is not extended. Met $84-\mathrm{C} \in$ lies at about the level of the front lip of the pocket, whereas Met $84-\mathrm{S} \gamma$ approaches Met 192 of the protease (C. Cambillau, pers. comm.). There appears to be ample room for an extended Met in the chymotrypsin pocket, so the structural basis for this crumpled conformation is unclear. The Met 84 side chain is out of the specificity pocket in collagenase (J.J. Perona, pers. comm.). Here, complete insertion into the pocket is clearly precluded by the major specificity determinant, Asp 226, which lies significantly higher up in the pocket than the analogous Asp 189 in trypsin. The collagenase pocket is also limited by intrusion of Thr 190 .

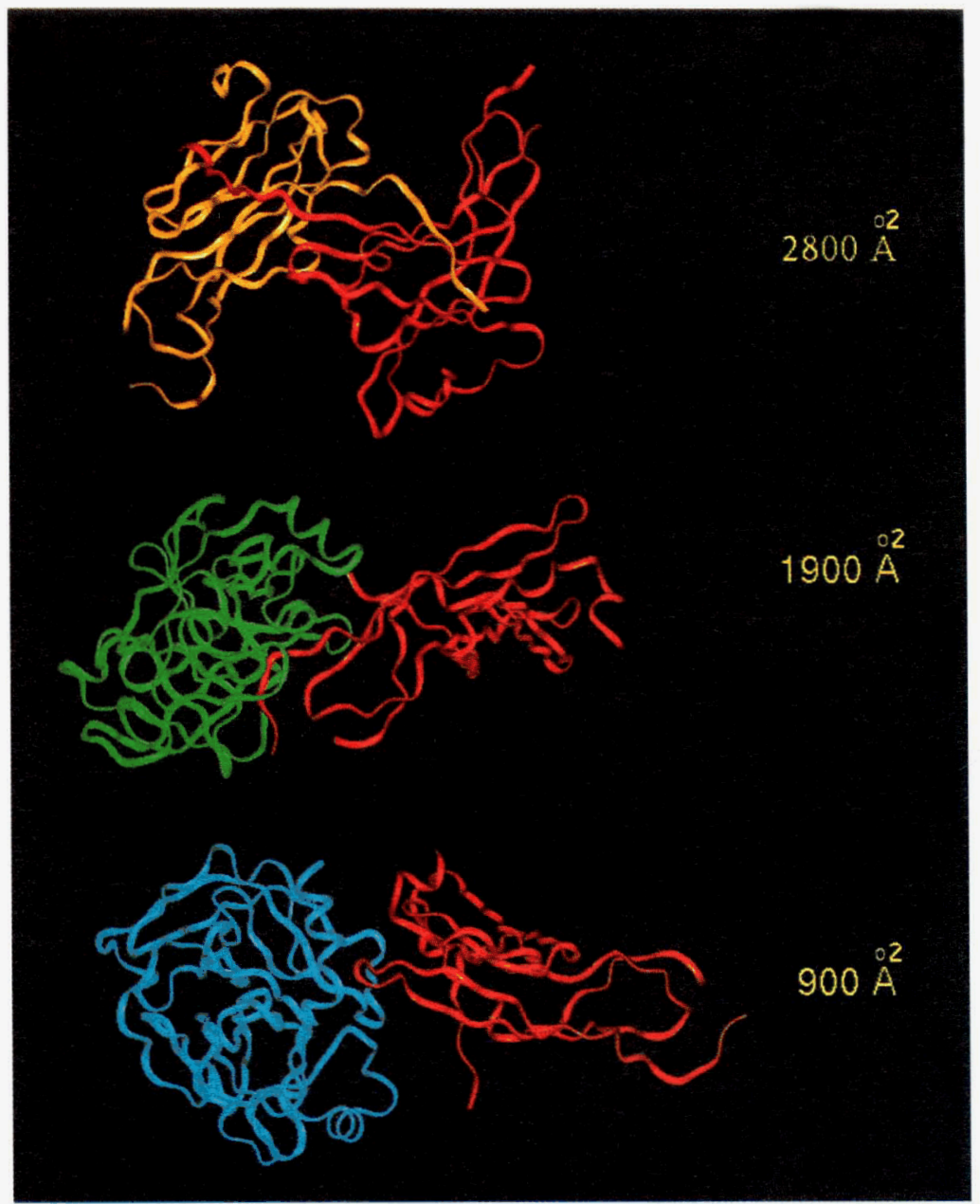

Fig. 3. Each ecotin monomer takes part in three distinct types of protein:protein interactions. The dimerization domain, as shown on top, buries a large area of the proteins' surfaces and is important for structural stability of the oligomer. The primary interaction site between ecotin and proteases utilizes turns and loops of the proteins. Ecotin's secondary binding site for proteases buries little surface area and would probably be unstable in the absence of the primary interaction. 


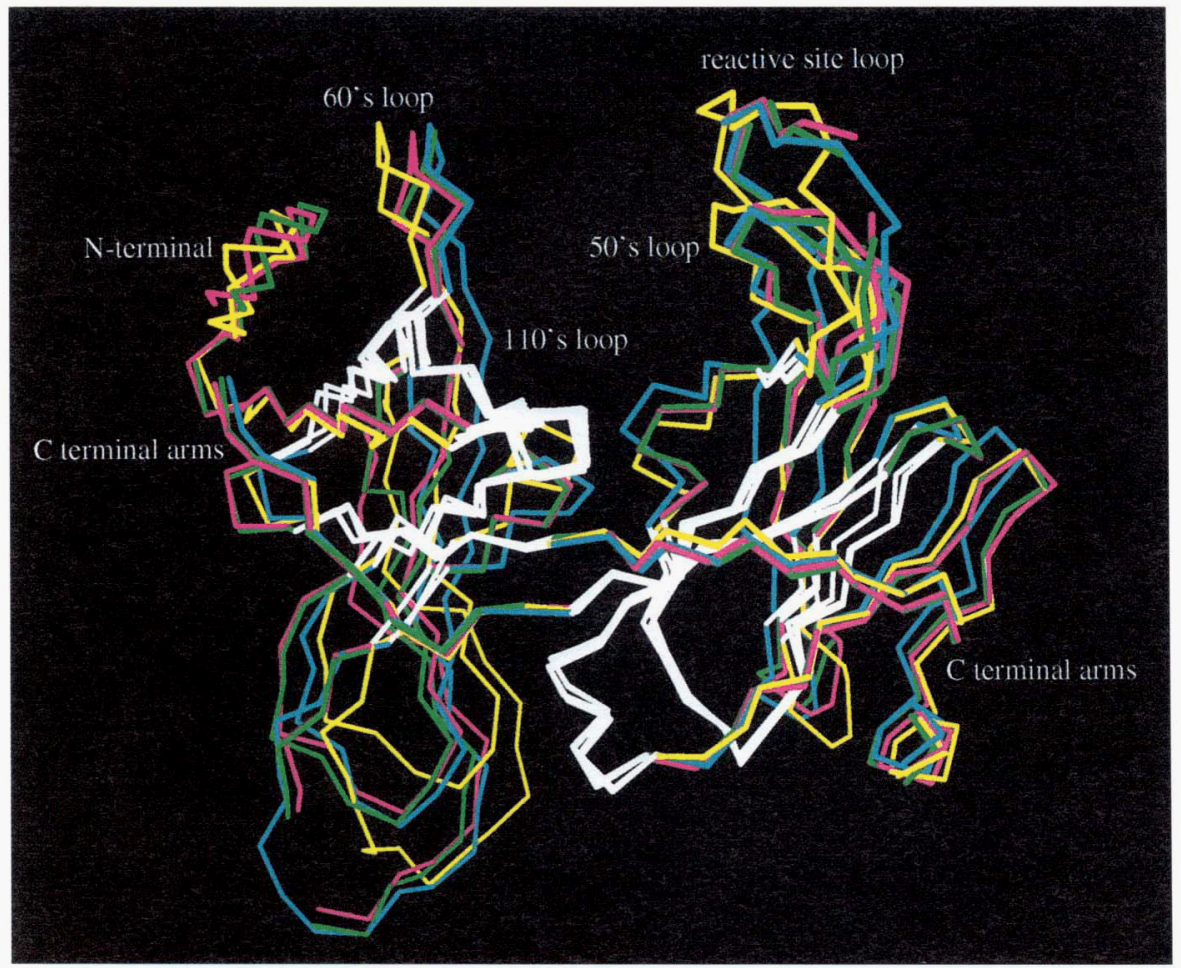

Fig. 4. Four structures for the ecotin dimer are shown as $\alpha$ carbon traces. Uncomplexed ecotin is yellow. The complex with collagenase is shown in blue; with trypsin, in red; and with chymotrypsin, in green. White bonds highlight a rigid core (RMS deviation $=0.4 \AA$ ) used to superimpose the dimers. The 110's loop that binds the proteases at the secondary site is rigid, unlike the other three identified loops.

The inherent flexibility of the Met side chain (Bernstein et al., 1989; O'Neil \& DeGrado, 1990) makes it perhaps the best choice for a panspecific inhibitor: it is able to contort so as to fit within or around the protease binding pocket. The $\mathrm{S} \gamma$ contributes to this flexibility (Gellman, 1991) as well as being a potential hydrogen bond acceptor (Gregoret et al., 1991).
Most inhibitors derive some binding energy from antiparallel $\beta$-strand interactions between the $\mathrm{P} 3$ region of the inhibitor and one side of the substrate binding pocket of the protease. In the complex with trypsin, for example, hydrogen bonds are found between ecotin Met 84-N (P1) and trypsin Ser 214-O, and between ecotin Ser 82-N and O (P3) with trypsin Gly 216-O and

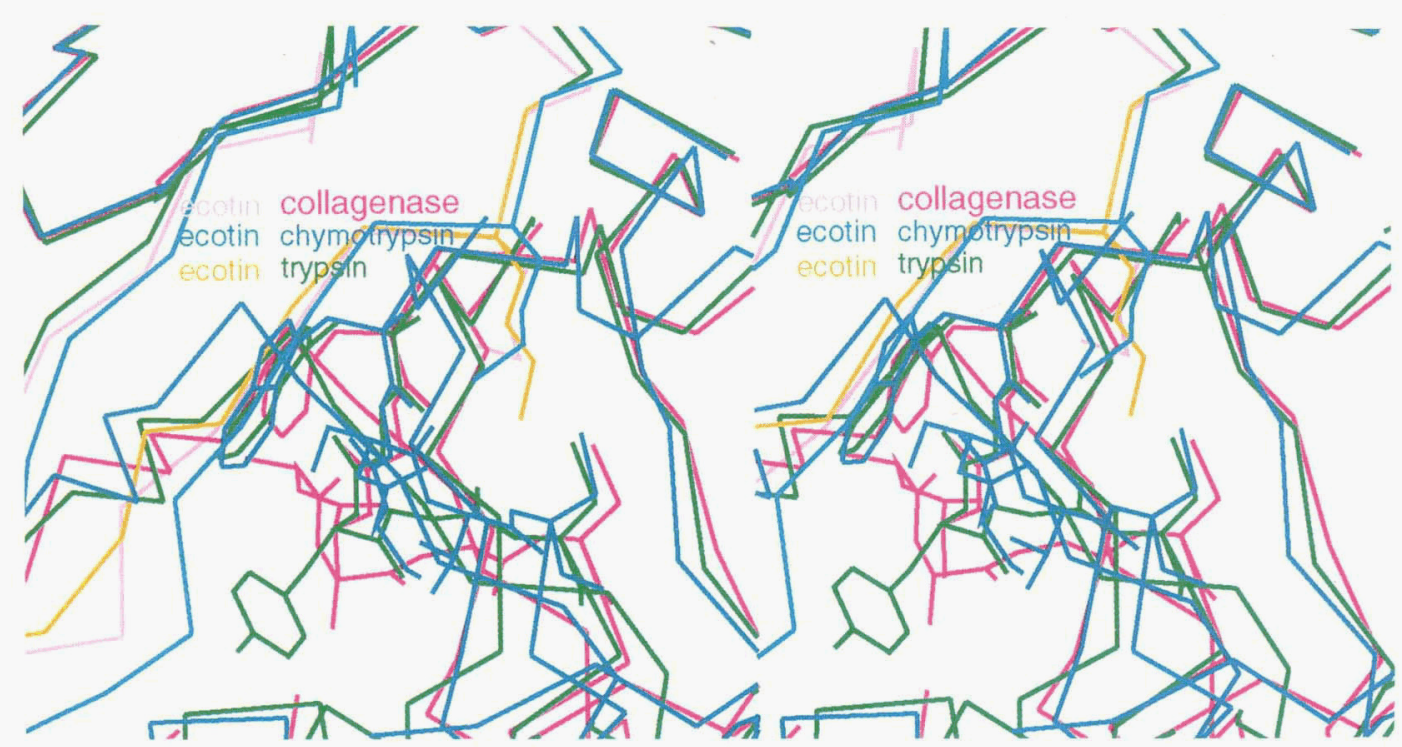

Fig. 5. Primary interaction sites for ecotin in its complexes with trypsin, chymotrypsin, and elastase are shown in stereo. In all cases, ecotin is shown as an $\alpha$ carbon trace, with only the side chain of Met 84 displayed. The proteases are likewise displayed, except that the residues which form the "front" of the enzymes' specificity pockets are shown. The conformational variability of the Met 84 side chain is obvious, as it adapts to the steric and charge characteristics of each binding pocket. 
$\mathrm{N}$, respectively. Additional interactions usually involve no more than the P4 to P3' segment of the reactive site loop. These typical contacts are seen for ecotin complexed with trypsin or chymotrypsin. A surprising find was the extent of this interaction with fiddler crab collagenase. Good hydrogen bonds exist between the protease and the 11-residue, P7 to P4', stretch of ecotin (residues 79-88) (J.J. Perona, pers. comm.). The additional contacts no doubt reveal part of the structural basis for collagenase's ability to cleave a triple helical substrate.

Additional contacts may be found between the inner supporting loop of the inhibitor and the protease. Whether by coincidence or design, Arg 39 provides these bonds in BPTI (Bode \& Schwager, 1975), whereas Arg 54 of ecotin extends to interact with the target protease. In the complexes with trypsin, chymotrypsin, and collagenase, Arg 54 forms one or more direct, or water-mediated, hydrogen bonds to the enzyme.

This primary interaction site between ecotin and protease utilizes loops and turns of the proteins and is a fairly typical functional interface that buries approximately $1,900 \AA^{2}$ of surface area (Fig. 3). (The range for a number of protease:inhibitor interactions is $1,325-2,050 \AA^{2}$.) Both the number of inhibitor residues involved ( 15 residues for the complex with trypsin) and the total number of interacting residues in the complex (34 residues) are in the expected ranges (Janin \& Chothia, 1990).

A

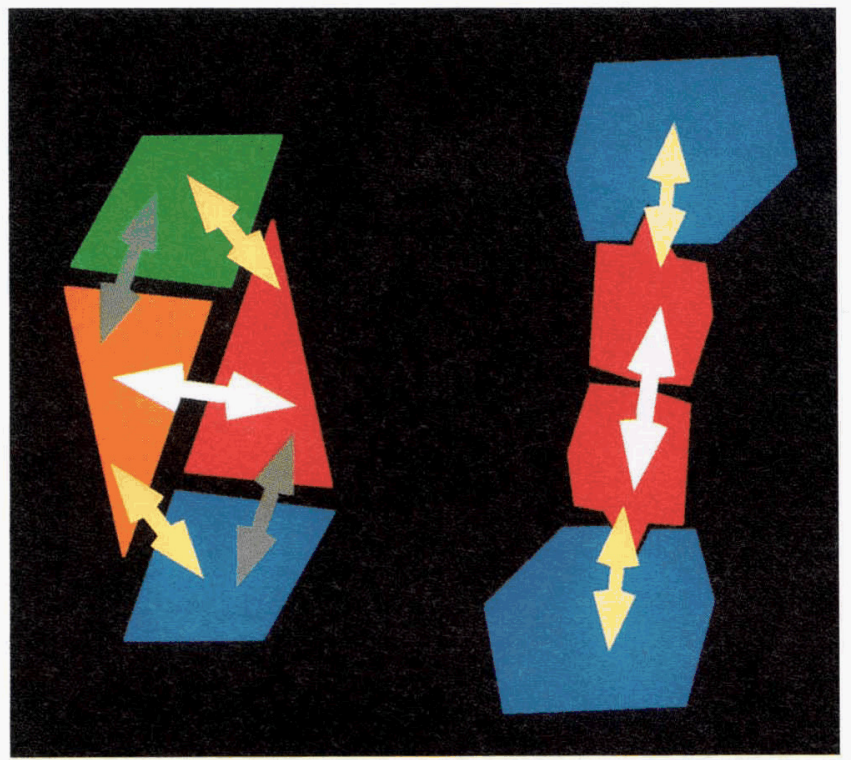

Fig. 6. A: "A network is stronger than its strongest link." The quaternary structure of ecotin in its complex with trypsin reveals a network structure that, by nature, strengthens the assembly. In contrast, the complex of dimeric Streptomyces subtilisin inhibitor and protease utilizes a linkage assembly in which each inhibitor contacts only one protease molecule. B: A space-filling representation of the ecotin:trypsin heterotetramer is also shown.

\section{Ecotin's secondary interactions with proteases}

Ecotin is different from all of the other serine protease inhibitors in having a generic secondary binding site for proteases. (The leech protein hirudin is a specific inhibitor of thrombin and interacts with a second locus on that protease [Rydel et al., 1990].) The site results from the dimer arrangement of ecotin. The dimer binds two protease molecules when available, and while one ecotin contacts a protease molecule's active site, the dyad-related ecotin's secondary site contacts a distal portion of that protease. Thus, in each heterotetramer, one ecotin contacts another ecotin and both trypsins.

SSI (Takeuchi et al., 1991) is another (and one of the few) protein inhibitors that is shown to bind two proteases. However, the SSI dimer interacts with each protease active site only through a reactive site on each monomer. The secondary ecotin/protease binding site, where ecotin contacts trypsin at a novel site using the dyad-related ecotin monomer, is a unique proteinprotease interaction and is unusual as a class of protein binding sites. In fact, ecotin:protease complexes are network-linked tetramers. Figure 6 shows a schematic of the tetramer network for the ecotin:protease complexes.

This secondary site is smaller than the primary site and, because of its small interaction surface of approximately $900 \AA^{2}$

B

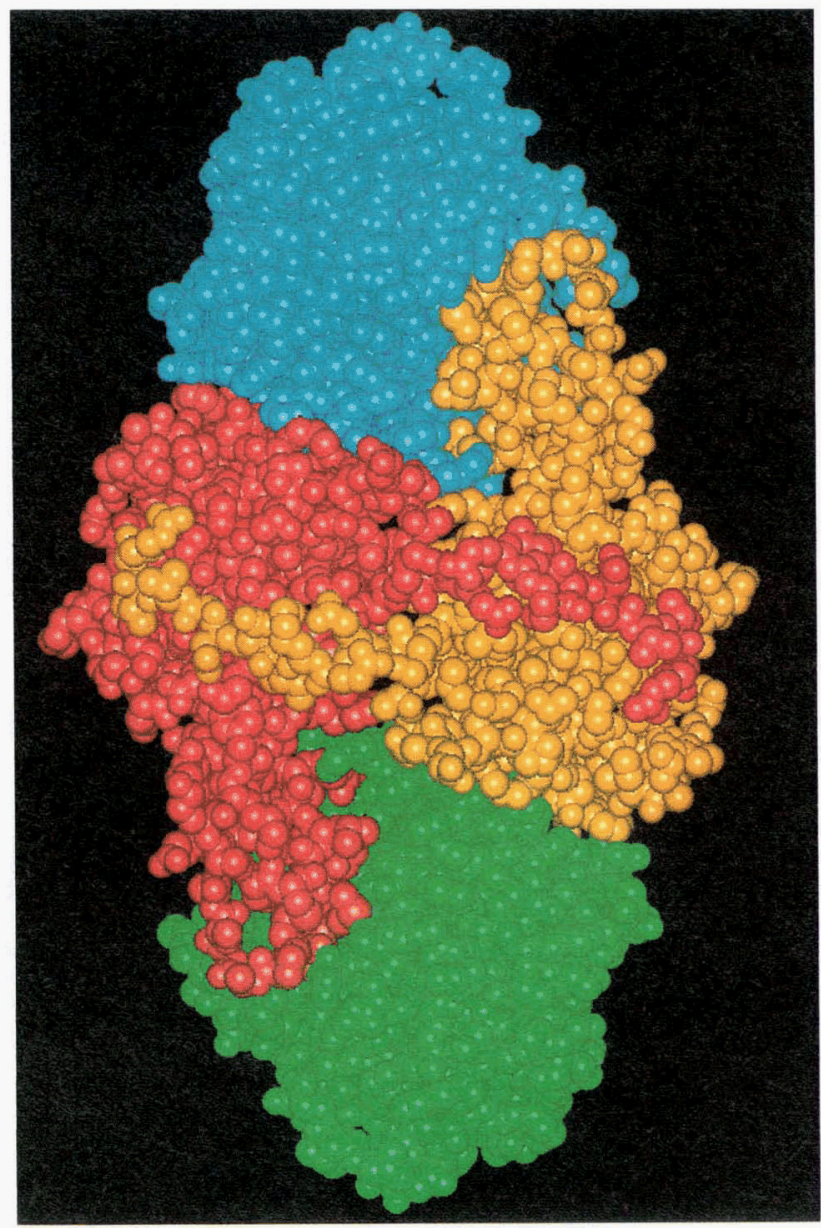


(Fig. 3), probably unstable in the absence of the primary interaction site. The secondary site is about half the usual size of a typical protein/protein contact site, but, surprisingly, it features a normal number of hydrogen bonds for such a site. For the ecotin:trypsin complex, nine direct hydrogen bonds and an additional four that are mediated by single water molecules stabilize this binding site. Very similar numbers and types of bonds are found in the complexes of ecotin with fiddler crab collagenase and chymotrypsin. In the latter complex, chymotrypsin's Gln 240 recruits additional residues from ecotin's $\mathrm{N}$-terminus for hydrogen bonding (C. Cambillau, pers. comm.).

The two interacting protein surfaces in the secondary site are flatter than that of the primary site. The shape of this site and geometric presentation of binding loops are reminiscent of those found in antibody combining sites (Janin \& Chothia, 1990). Specifically, the 60's and 110's loops of ecotin are topologically and functionally analogous (though differing in chain polarity) to the complementarity determining regions (CDR) of an antibody (Fig. 7). In an IgG antibody, the CDR2 and CDR3 loops both connect adjacent strands of a single $\beta$-sheet, much like the ecotin 60's loop. In the antibody, the CDR1 loop is longer and connects strands on opposite sides of the two $\beta$-sheets, like the ecotin 107-114 loop (Brändén \& Tooze, 1991). The ecotin dimer, like an antibody, uses two $\beta$-sandwiches to bind a protein (Chothia \& Lesk, 1987), but only for the interactions in the secondary site. This juxtaposition of two $\beta$-sandwiches and topological arrangement of loops is apparently a good framework to bind proteins.

The combination of substrate-like primary site and antibodylike secondary site may allow ecotin to tightly bind a wider range of proteases than a substrate mimic alone because primary site interactions that are less than optimal can be compensated for by the additional secondary site interactions. Even with several ecotin:protease structures available, it is surprising that the sec-

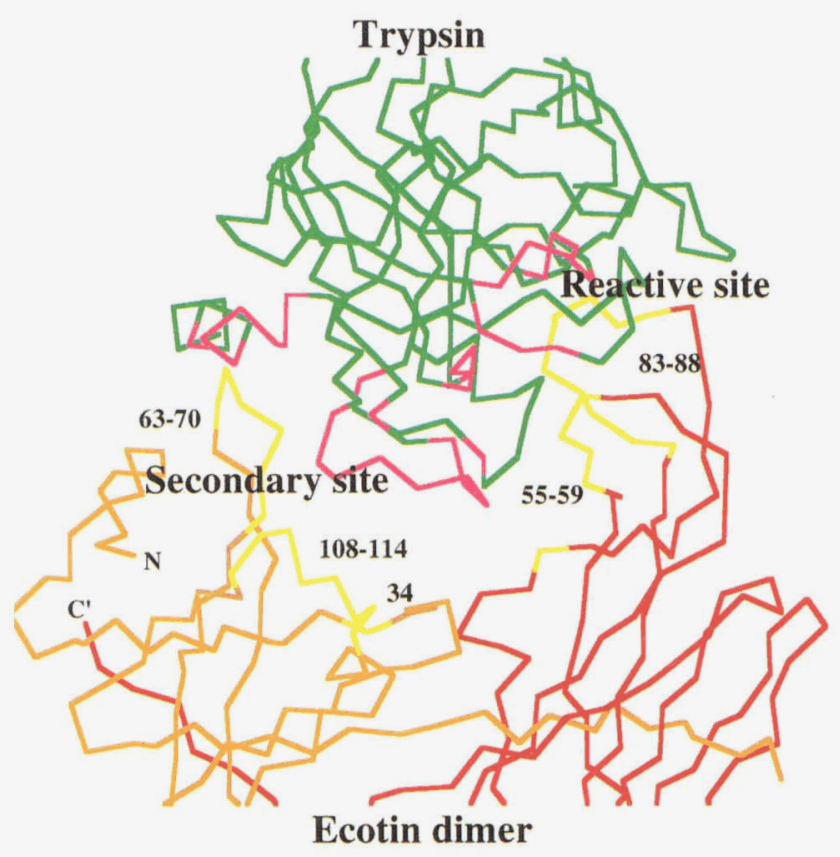

Fig. 7. Loops that comprise the secondary binding site of ecotin for protease are shown and compared with the structurally similar loops utilized in antibody combining sites. ondary site of ecotin binds a family of proteases that show sequence variability at the secondary site. Pliability of dimeric ecotin undoubtedly contributes to this phenomenon. The essential elements of this flexibility are likely to reside in the structurally distinct positioning of ecotin's primary and secondary binding loops, the network-like arrangement of the quaternary structure, and the potential to employ solvent to fill in the divots at the proteins' interface.

\section{What's next for ecotin?}

The initial lack of interest in ecotin has been replaced by an enthusiastic desire to understand this inhibitor. Several laboratories have embarked on mutagenesis studies that promise to dissect out the relative importance of the primary and secondary sites, and that will delineate the boundaries of how general and how specific ecotin can be made in its inhibitory capacity (C.S. Craik, pers. comm.; Lauwereys et al., 1993; Pal et al., 1994; Seong et al., 1994). One highlight from this work is an ecotin designed to inhibit thrombin (ecotin Met 84 Arg) against which the natural inhibitor is feckless (Lauwereys et al., 1993). Another is an exciting study in which ecotin has been successfully displayed on phage. The results show that the inhibitor's reactive site can be redesigned for increased potency against specific target proteases (C.S. Craik, pers. comm.). We also expect to see additional high-resolution structures of ecotin complexes. These will, no doubt, provide more information and, as always, more questions.

\section{Acknowledgments}

This work was partially supported by NIH DK39304 to R.J.F. and an NSF predoctoral fellowship to S.A.G. We thank Drs. Christian Cambillau, SeWon Suh, John Perona, and Charles Craik for providing X-ray coordinates or other experimental data prior to publication.

\section{References}

Baker D, Bystroff C, Fletterick RJ, Agard DA. 1993. PRISM: Topologically constrained phase refinement for macromolecular crystallography. Acta Crystallogr D 49:439-448.

Bernstein HD, Poritz MA, Strub K, Hoben PJ, Brenner S, Walter P. 1989. Model for signal sequence recognition from amino acid sequence of $54 \mathrm{~K}$ subunit of signal recognition particle. Nature 340:482-486.

Bode W, Greyling HJ, Huber R, Otlewski J, Wilusz T. 1989. The refined 2.0 angstrom X-ray crystal structure of the complex formed between bovine beta-trypsin and CMTI-1, a trypsin inhibitor from squash seeds (Cucurbita maxima): Topological similarity of the squash seed inhibitors with the carboxypeptidase A inhibitor from potatoes. FEBS Lett 242:285-292.

Bode W, Huber R. 1992. Natural protein proteinase inhibitors and their interactions with proteinases. Eur $J$ Biochem 204:433-451.

Bode W, Schwager P. 1975. The refined crystal structure of bovine $\beta$-trypsin at $1.8 \AA$ resolution. $J \mathrm{Mol}$ Biol 98:693-717.

Brändén CI, Tooze J. 1991. Introduction to protein structure. New York: Garland Publishing, Inc.

Brünger AT. 1990. Extension of molecular replacement: A new search strategy based on Patterson correlation refinement. Acta Crystallogr A 46:46-57.

Bystroff C, Baker D, Fletterick RJ, Agard DA. 1993. PRISM: Application to the solution of two protein structures. Acta Crystallogr D 49:429-439.

Chen Z, Bode W. 1983. Refined $2.5 \AA$ X-ray crystal structure of the complex formed by porcine kallikrein $\mathrm{A}$ and the bovine pancreatic trypsin inhibitor. Crystallization, Patterson search, structure determination, refinement, structure and comparison with its components and with the bovine trypsin:pancreatic trypsin inhibitor complex. $J \mathrm{Mol} \mathrm{Biol} \mathrm{164:}$ 283-311.

Chothia C, Lesk A. 1987. Canonical structures for the hypervariable regions of immunoglobulins. J Mol Biol 196:901-917. 
Chung CH, Ives HE, Almeda S, Goldberg AL. 1983. Purification from Esch erichia coli of a periplasmic protein that is a potent inhibitor of pancre atic proteases. I Biol Chem 258:11032-11038.

Erpel T, Hwang P, Craik CS, Fletterick RJ, McGrath ME. 1992. Physica map location of the new Escherichia coli gene eco, encoding the serine protease inhibitor ecotin. $J$ Bacteriol 174:1704.

Fujinaga M, Sielecki AR, Read RJ, Ardelt W, Laskowski M Jr, James MN. 1987. Crystal and molecular structures of the complex of alpha-chymo trypsin with its inhibitor turkey ovomucoid third domain at $1.8 \AA$ resolution. J Mol Biol 195:397-418.

Gellman SH. 1991. On the role of methionine residues in the sequenceindependent recognition of nonpolar protein surfaces. Biochemistry 30 6633-6636.

Gregoret L, Rader SD, Fletterick RJ, Cohen FE. 1991. Hydrogen bonds involving sulfur atoms in proteins. Proteins Struct Funct Genet 9:99-107.

Henderson R, Wright C, Hess G, Blow D. 1972. $\alpha$-Chymotrypsin: What can we learn about catalysis from X-ray diffraction? Cold Spring Harbor Symp Quant Biol 36:63-70.

Huang K, Strynadka NCJ, Bernard VD, Peanasky RJ, James MNG. 1994 The molecular structure of the complex of Ascaris chymotrypsin/elastase inhibitor with porcine elastase. Structure 2:679-689.

Janin J, Chothia C. 1990. The structure of protein-protein recognition sites $J$ Biol Chem 265:16027-16030.

Laskowski M, Kato I. 1980. Protein inhibitors of proteinases. Annu Rev Biochem 49:593-626.

Lauwereys M, Stanssens P, Lambier AM, Messens J, Dempsey E, Vlasuk GP. 1993. Ecotin as a potent factor Xa inhibitor. Thromb Haemostasis $69: 864$

Lee HR, Seo JH, Kim OM, Lee CS, Suh SW, Hong YM, Tanaka K, Ichihara A, Ha DB, Chung CH. 1991. Molecular cloning of the ecotin gene in Escherichia coli. FEBS Lett 287:53-56.

McGrath ME, Engel T, Bystroff C, Fletterick RJ, 1994. Macromolecular chelation as an improved mechanism of protease inhibition: Structure of the ecotin-trypsin complex. EMBO J 13:1502-1507.

McGrath ME, Erpel T, Browner MF, Fletterick RJ. 1991a. Expression of the protease inhibitor ecotin and its co-crystallization with trypsin. $J \mathrm{Mol}$ Biol 222:139-142.
McGrath ME, Hines WM, Sakanari JA, Fletterick RJ, Craik CS. 1991b. The sequence and reactive site of ecotin. J Biol Chem 266:6620-6625.

O'Neil KT, DeGrado WF. 1990 . How calmodulin binds its targets: Sequence independent recognition of amphilic alpha-helices. Trends Biochem Sci $15: 59-64$.

Pal G, Sprengel G, Patthy A, Graf L. 1994. Alteration of the specificity of ecotin, an $E$. coli serine proteinase inhibitor, by site directed mutagenesis. FEBS Lett 342:57-60.

Perona JJ, Tsu C, Fletterick RJ, Craik CS. 1993. Crystal structures of rat anionic trypsin complexed with the protein inhibitors APPI and BPTI. I Mol Biol 230:934-949.

Ruhlmann A, Kukla D, Schwager P, Bartels K, Huber R. 1973. Structure of the complex formed by bovine trypsin and bovine pancreatic trypsin inhibitor. $J$ Mol Biol 77:417-436.

Rydel TJ, Ravichandran KG, Tulinsky A, Bode W, Huber R, Roitsch C, Fenton JW. 1990. The structure of a complex of recombinant hirudin and human alpha-thrombin. Science 249:277-280.

Seong IS, Lee HR, Seol JH, Park SK, Lee CS, Suh SW, Hong YM, Ha DB, Chung $\mathrm{CH}$. 1994. The $\mathrm{Pl}$ reactive site methionine residue of ecotin is not crucial for its specificity on target proteases. $J$ Biol Chem 269: 21915-21918.

Seymour JL, Lindquist RN, Dennis MS, Moffat B, Yansura D, Reilly D, Wes singer ME, Lazarus RA. 1994. Ecotin is a potent anticoagulant and reversible tight-binding inhibitor of factor Xa. Biochemistry 33:3949-3958.

Shin DH, Hwang KY, Kim KK, Lee HR, Lee CS, Chung CH, Suh SW. 1993. Crystallization and preliminary X-ray crystallographic analysis of the protease inhibitor ecotin. J Mol Biol 229:1157-1158.

Sweet RM, Wright HT, Janin J, Chothia CH, Blow DM. 1974. Crystal structure of the complex of porcine trypsin with soybean trypsin inhibitor (Kunitz) at 2.6 A resolution. Biochemistry 13:4212-4228.

Takeuchi Y, Satow Y, Nakamura KT, Mitsui Y. 1991. Refined crystal structure of the complex of subtilisin BPN' and Streptomyces subtilisin inhibitor at $1.8 \AA$ resolution. $J$ Mol Biol 22I:309-325.

Tsu C, Perona JJ, Schellenberger V, Turck CW, Craik CS. 1994. The substrate specificity of Uca pugilator collagenolytic serine protease 1 correlates with the bovine type I collagen cleavage sites. $J$ Biol Chem 269:19565-19572. 